\title{
ADVANCES IN LARGE GRAIN RESONATORS FOR THE EUROPEAN XFEL
}

\author{
W. Singer, S. Aderhold, J. Iversen, G. Kreps, A. Matheisen, X. Singer, K. Twarowski, H. Weise \\ (DESY) \\ M. Pekeler (Research Instruments GmbH) \\ F. Schölz, B. Spaniol, E. Stiedl (W.C. HERAEUS)
}

\begin{abstract}
An overview of the activities within the DESY test program of $1.3 \mathrm{GHz}$ TESLA shape 9-cell Large Grain (LG) resonators for the European XFEL, which have taken place in last 4 years, is presented. Attention is devoted to development of LG disc production and cavity fabrication from this material, focusing in particular on aspects of production at reasonable accuracy and costs. More than 200 LG discs were manufactured, eleven 9-cell resonators produced, partially treated at the company Research Instruments (RI) (former ACCEL) and finally treated and RF tested at DESY. Two of the LG cavities are currently used in the FLASH accelerator operation, which is the best demonstration of the feasibility of the LG application.

The program compares large grain material with standard sheet niobium. Some data and perspectives of the LG application are discussed.
\end{abstract}

\section{INTRODUCTION}

The manufacturing approach of slicing discs from the melted ingot and producing cavities by deep drawing and electron beam welding (LG cavities) has been proposed by Jefferson Laboratory (Jlab) and CBMM [1]. This option allows for the elimination of the long production chain from large grain ingot to fine grain sheet and seems to be cost effective assuming that the slicing can be done by keeping the material purity high and achieving tight thickness tolerances and high surface quality. It can be expected that disc material from ingots is less vulnerable to contamination with foreign material or other types of defects, because it is taken directly from ingot material that has been remelted many times. Several sources of contamination that occur during forging or rolling in the standard procedure of producing conventional sheets are excluded in this case. It has also been shown that large grain and single crystal high pressure water rinsed $\mathrm{Nb}$ samples provide less field emission than polycrystalline ones [12].

During the last years several single cell cavities were produced at Jlab, DESY and IHEP/KEK from large grain high purity niobium. Very encouraging results were demonstrated after buffered chemical polishing (BCP) treatment or electropolishing (EP) [2-4 ].

The main aim of the DESY R\&D program is to check to what extent the 9-cell LG cavities can be built for and applied to particle accelerators like the European XFEL and others.

Several aspects of LG cavities have been investigated. One of the aspects is the material issue: could LG niobium be produced by industry in a cost effective and reliable manner? The second aspect is the fabrication of cavities: can the production of 9-cell LG resonators be done at the level of required accuracy and costs? The third one is the performance issue: what will be the highest achieved accelerating gradient, what is the appropriate treatment for achieving a stable and reproducible gradient, do LG cavities have advantages besides costs comparable to fine grain material cavities? 


\section{MATERIAL}

Development of LG disc production was done within the framework of the R\&D program of DESY and the company W. C. HERAEUS in 2007-2008.

One of the DESY requirements was the presence of a single crystal with a diameter larger than 150 $\mathrm{mm}$ in the disc's central area. On one hand this demand is essential for avoiding necking and tearing at the irises during deep drawing, on the other hand it should provide field emission reduction, which could be expected on large-grain boundary steps in the area with an enhanced electrical field. In order to achieve cost effective production, a single crystal with these dimensions has to be continuously present in the entire, approx. two meters long ingot. The company investigated the impact of different melting parameters on the peculiarities of solidification and crystal growth. A lot of attention concerns the melting-/cooling behavior: beam figures (different numbers, position und shape); energy entry (different focusing of the beam and stay time); refrigeration parameters (bottom, crucible wall, split) etc.

Several times LG ingots have been successfully produced to target specifications. Large grain crystal grows longitudinally (see Fig.1 for example). Material for several dozens of cavities was produced. On the other hand, it turned out that production of the specified big central crystal, especially with the desired crystal orientation, is very critical and at the moment cannot be achieved over the complete length of the ingot.

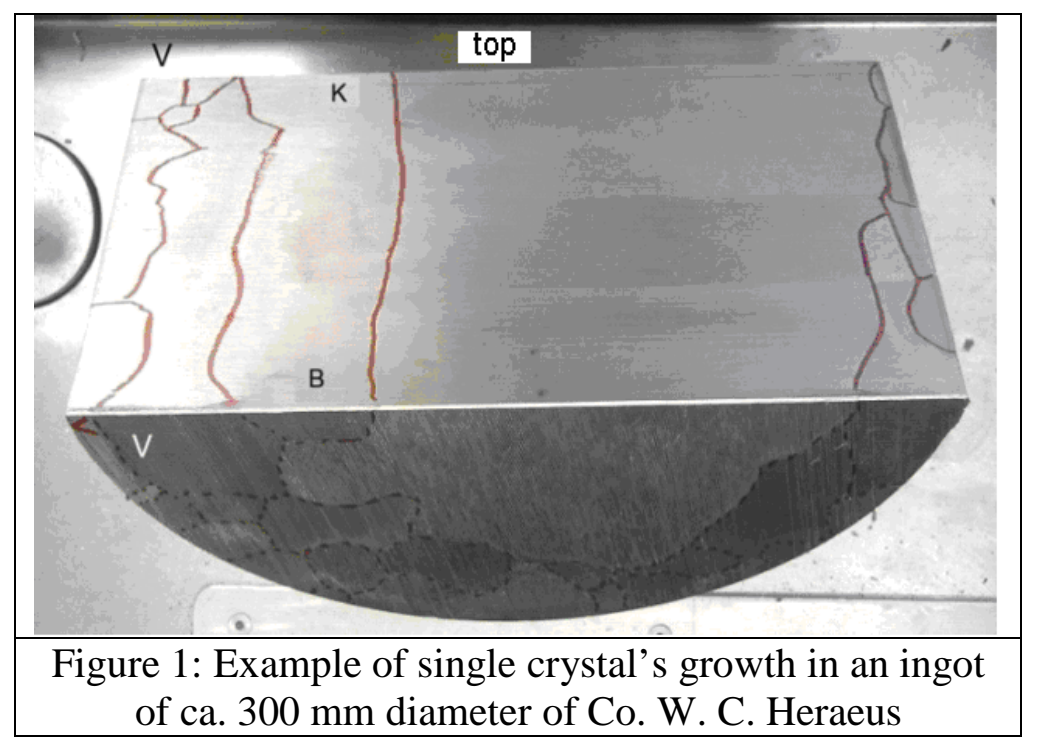

The efforts towards creating a central crystal along the whole ingot in a stable and reproducible manner will continue.

The second requirement was cost-effectively cutting the discs with tight thickness tolerances, purity and high surface quality for cavity half cells. The discs applied for single cell cavity fabrications were produced by EDM slicing or by combination of sawing and surface finishing. Such an expensive procedure is not usable for mass production. To this aim W.C. HERAEUS developed the multi-wire slicing procedure based on silicon wafer technology to the extent that disc slicing can be done without degrading the material purity. 


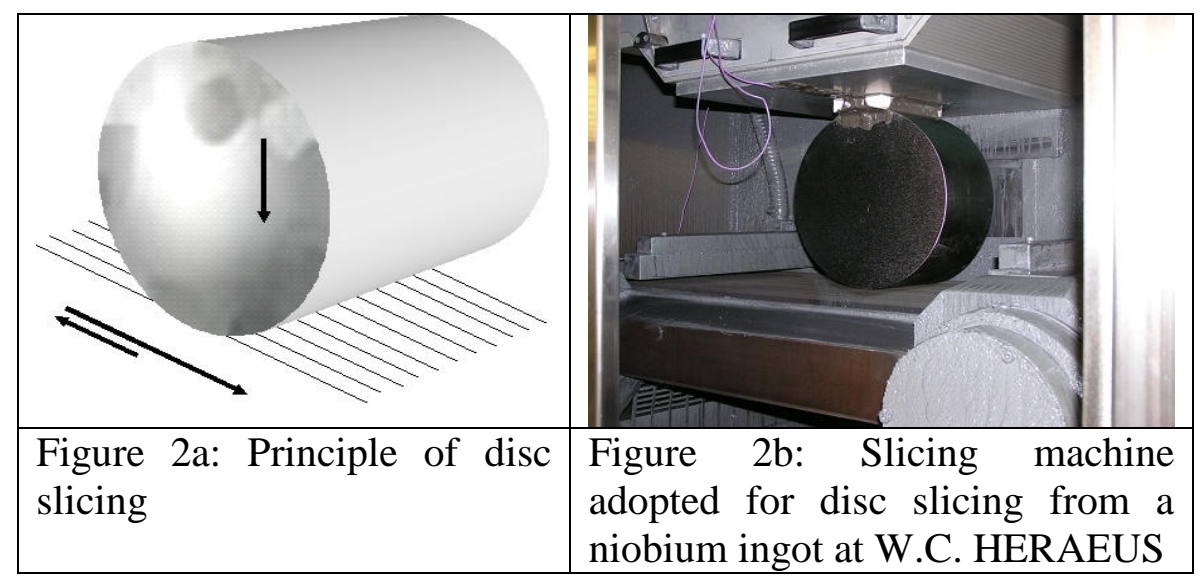

The slicing principle and the slicing machine adopted at W.C. HERAEUS for disc slicing from a niobium ingot can be seen in Fig. $2 \mathrm{a} \& \mathrm{~b}$. An ingot is pressed on the multi-wire. The wire is moving forwards and backwards; liquid abrasives are sprayed on the wire. The developed procedure allows keeping the high $R R R$ values $(R R R=300$ - 500) of the melted high purity ingot in the discs. KEK, Tokyo Denkai, and TKX Corporation have now developed a similar slicing technique [4], the slicing machine is installed at the company Tokyo Denkai.

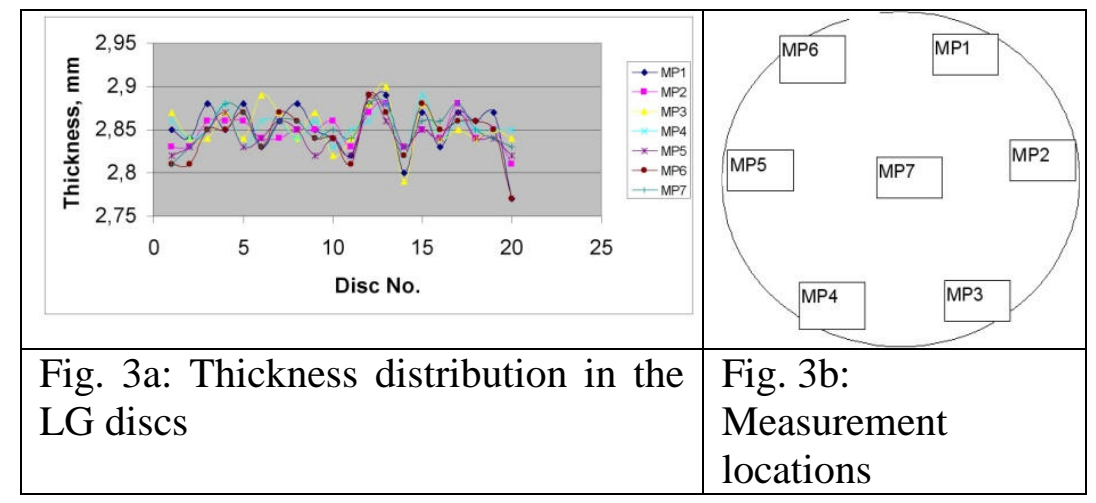

Figure 3a shows as an example the average thickness of the 20 sliced LG discs. The thickness measurement was done on seven locations per disc (Fig. 3b). In all cases the thickness of the sliced LG discs meets the requirements of the material specification $(2.8+/-0.1 \mathrm{~mm})$. The surface of the sliced discs has a high quality, roughness $\mathrm{Ra}$ was within of $0.3-0.6 \mu \mathrm{m}$, which is better than specified $(\mathrm{Ra}<1.6 \mu \mathrm{m})$.

The benefits of slicing method in terms of simplified production procedure and in terms of lower material costs are described in detail in reference [4] and will not be discussed here.

Approximately 250 large grain niobium discs with $R R R=320-500$ and different crystal orientation of the central grain have been sliced at W.C. HERAEUS and shipped to DESY. The main discs used for manufacturing of the first three 9-cell cavities were scanned with an eddy current device available at DESY. As expected no indications of localized foreign material inclusions in the scanned area were observed (see [5]). Therefore, it was decided not to scan the discs intended for the next 8 LG cavities.

\section{FABRICATION}

Eleven LG cavities AC112-AC114 and AC151-AC158 of TESLA shape have been produced at the company ACCEL (now RI), from W.C. HERAEUS material. The tools created for fine grain 
material have been applied for deep drawing of the half cells. The grain boundaries were noticeably pronounced with steps up to $0.5 \mathrm{~mm}$ (Fig. 4a). In two cavities, AC155 and AC156, the steps on grain boundaries have been grinded away with the intention of comparing the performance of ground and non-ground cavities.

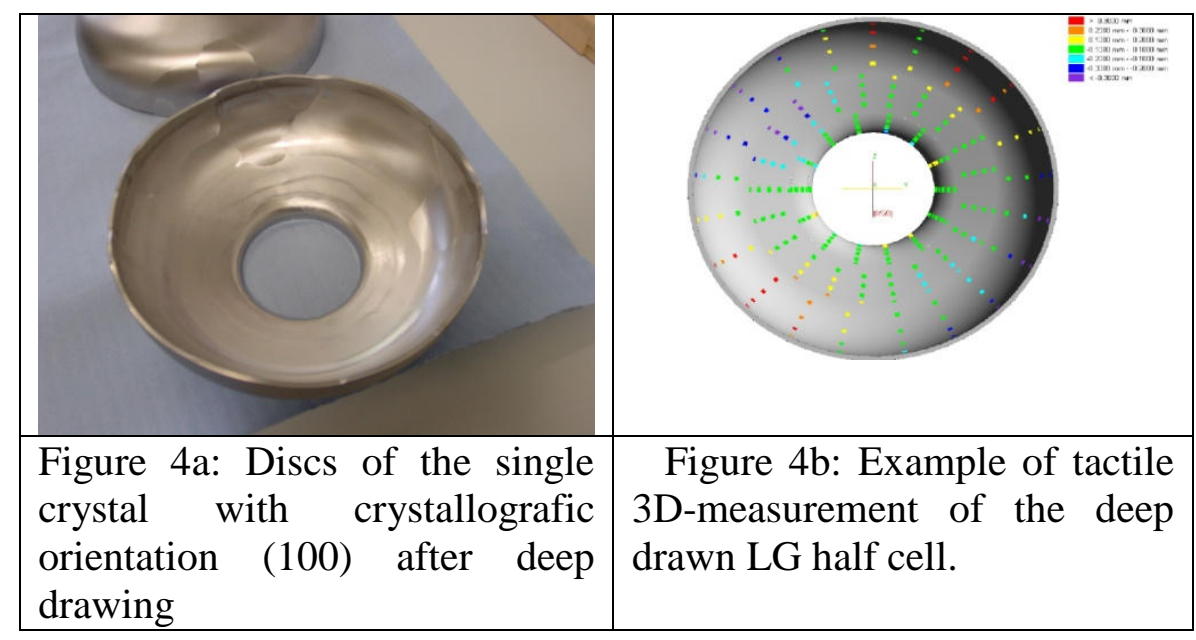

Tactile 3D measurement at ACCEL and optical coordinate measurement with 3D imaging at the company DECOM was applied in order to estimate the shape accuracy. In most cases the accuracy of the shape conformed with specification, however some half cells have a quadrangular or oval shape and were out of tolerance (tolerance: $+/-0.2 \mathrm{~mm}$ ) (see also figure $5 \mathrm{a} \& \mathrm{~b}$ ).

It was observed that the half cells' shape deviation for cavities AC151-AC154 was very pronounced compared to cavities AC155-AC158. It turned out that this is essentially due to the difference of the orientation of central crystals in the discs.

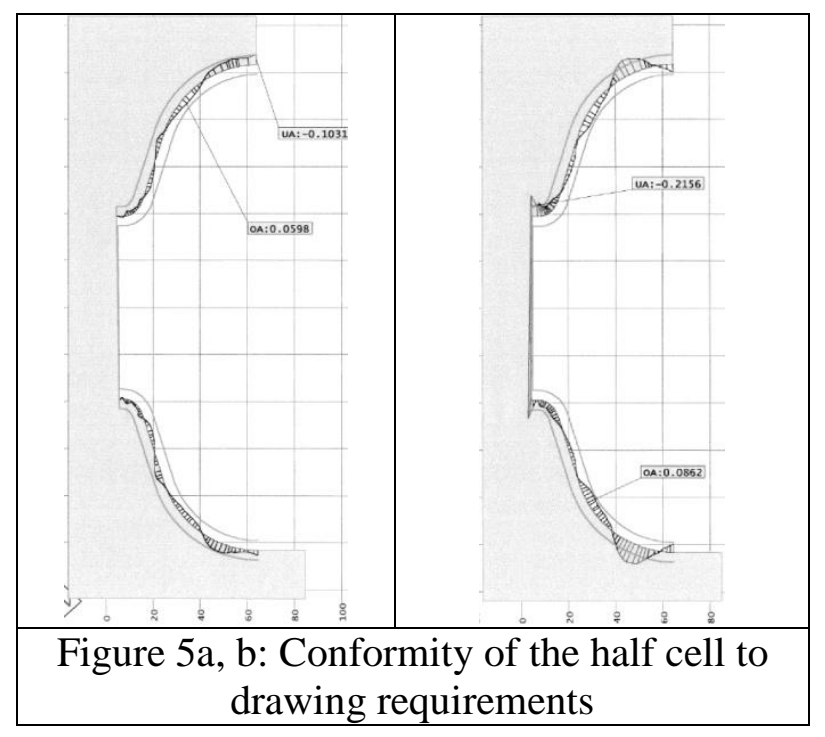

Analysis of the crystal orientation done with the Lauer technique showed that in discs AC151AC154, the main orientation of the central crystal is (100), and for discs of AC155-AC158, it was mainly (211) or (221). It is well known that the slipping of the main atom planes for BCC metals takes place in the (110) planes in the [111] direction; therefore, for the dics with (100) orientation, a more prononced anisotropy and quadrangular shape after deep drawing should be expected. This 
can be easily seen in figure 6, where the averaged deviation from the ideal shape of the half cell for different crystal orientations is shown. The (211) or (221) planes tend to be more oval shaped and represent smaller anisotropy.

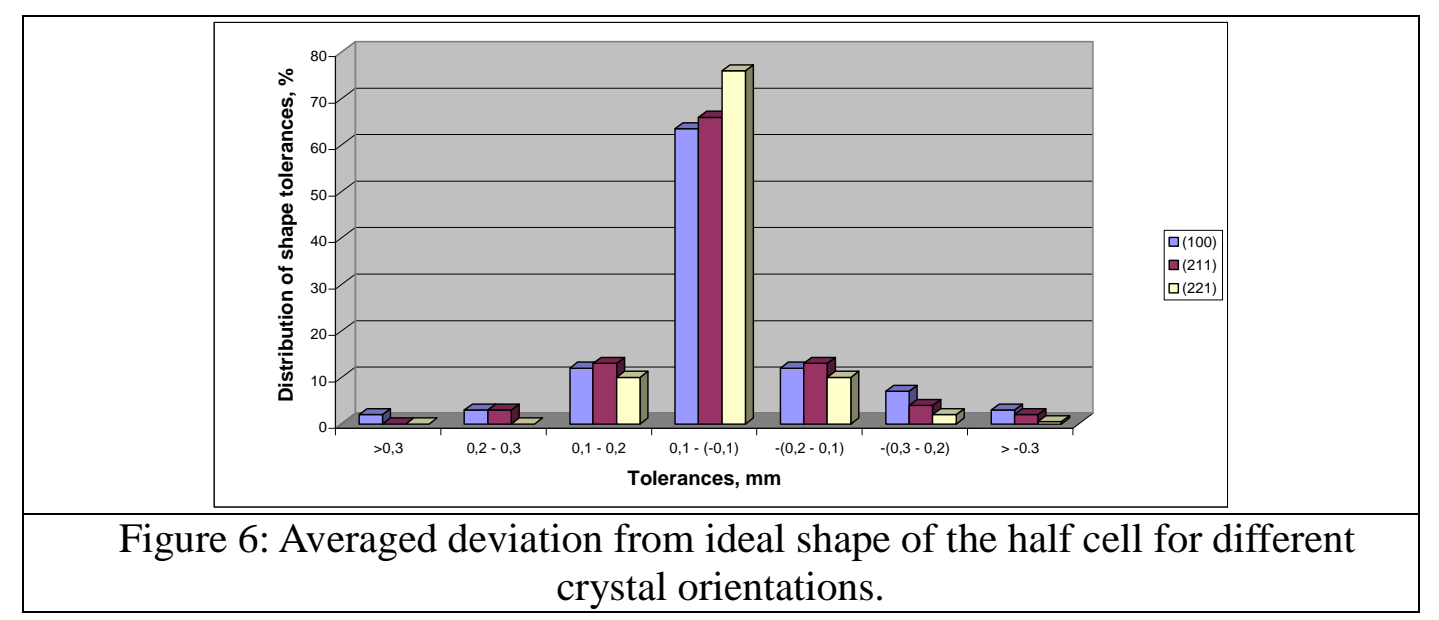

The pronounced shape deviation in the half cells generated some difficulties for assembling half cells into dumb-bells for welding. These difficulties have been overcome by using a special tool that ensures precise assembly of the male and female half cells together. Applying the length adjustment procedure [6] developed at DESY helped to achieve the correct cavity length and frequency of the fundamental mode in all LG cavities AC112-AC114 and AC151-AC158.

\section{PREPARATION AND RF TESTS}

Investigations on single cell cavities [2] have shown that relatively good performance can be achieved by rather simple $\mathrm{BCP}$ treatment $\mathrm{HF}(40 \%): \mathrm{HNO}_{3}:(65 \%) \mathrm{H}_{3} \mathrm{PO}_{4}(85 \%)$ at the volume ratio 1:1:2 available in most laboratories. Therefore, as a first step, a BCP treatment consisting of about $100 \mu \mathrm{m}$ rough $\mathrm{BCP}$, annealing at $800^{\circ} \mathrm{C}$ for $2 \mathrm{~h}$ and $20 \mu \mathrm{m}$ fine $\mathrm{BCP}$ was done on first three cavities AC112-AC114. Already after the first surface treatment, the specification for the XFEL, namely Eacc $=24.3 \mathrm{MV} / \mathrm{m}$ with a quality factor $\mathrm{Q}=1 \times 10^{10}$, was exceeded. A second test was done on two cavities (AC113 and AC114) after additional $20 \mu \mathrm{m}$ fine $\mathrm{BCP}$ and baking at $125^{\circ} \mathrm{C}$ for $48 \mathrm{~h}$. After insitu baking, the Q-drop almost disappeared, but the accelerating gradient was not increased [3, 7].

Unfortunately, because of the very tight schedule of the XFEL preparation phase it was not possible to carry out a comprehensive treatment and RF testing program. Therefore the following treatment was applied to cavities AC151-AC158: $100 \mu \mathrm{m}$ rough $\mathrm{BCP}$, annealing at $800^{\circ} \mathrm{C}$ for $2 \mathrm{~h}$, fine $\mathrm{BCP}$ of $20 \mu \mathrm{m}$ followed by baking at $125^{\circ} \mathrm{C}$ for $48 \mathrm{~h}$ (only $\mathrm{AC} 112$ was not baked). While the rough BCP treatment (removal of $100 \mu \mathrm{m}$ inside and $20 \mu \mathrm{m}$ outside) for AC112-AC114 was done at DESY, the treatment for cavities AC151-AC158 was done at the company ACCEL (now RI). The fine $\mathrm{BCP}$ procedure and remaining treatment were carried out at DESY.

The $\mathrm{Q}$ (Eacc) excitation curves, in most cases measured after first treatment, can be seen in Fig. 7. Unfortunately the performance of some cavities (in particular AC156, AC152, AC158) was partially restricted by field emission after first treatment. Thus additional HPR was done on cavities AC156 and AC152 which significantly improved the performance; here the second test result is shown in Fig. 7 for the respective cavities. The performance of most of the cavities was limited by quench. After in-situ baking, the Q-drop at high gradients (if not affected by field emission) almost disappeared in agreement with the observed behavior on single cell BCP-treated and baked LG cavities [8]. This result is to be compared with the well-known fact that for BCP treated fine-grain 
cavities baking almost does not change the Qo-drop. Eacc up to $35 \mathrm{MV} / \mathrm{m}$ was achieved in the pass band measurement.

The achieved accelerating gradients between 25 and $30 \mathrm{MV} / \mathrm{m}$ in the $\pi$-mode measurement (which corresponds to the average quench magnetic field Bp of 110-130 mT) in the LG TESLA shaped cavities can be considered a very good result for XFEL. Already after the first surface treatment, the specification for the XFEL, Eacc $=24.3 \mathrm{MV} / \mathrm{m}$ with a quality factor $\mathrm{Q}=1 \times 10^{10}$, was in most cases exceeded. Out of the group of eight cavities AC151 - AC158, only one cavity (AC158), restricted by enhanced field emission, barely fulfilled the requirements. As mentioned above, the steps on grain boundaries have been grinded on two cavities AC155 and AC156. Both grinded cavities do not show any deviation compared to other cavities. This means that LG cavities did not show any indication that the grinding of grain boundary steps leads to a benefit in performance.

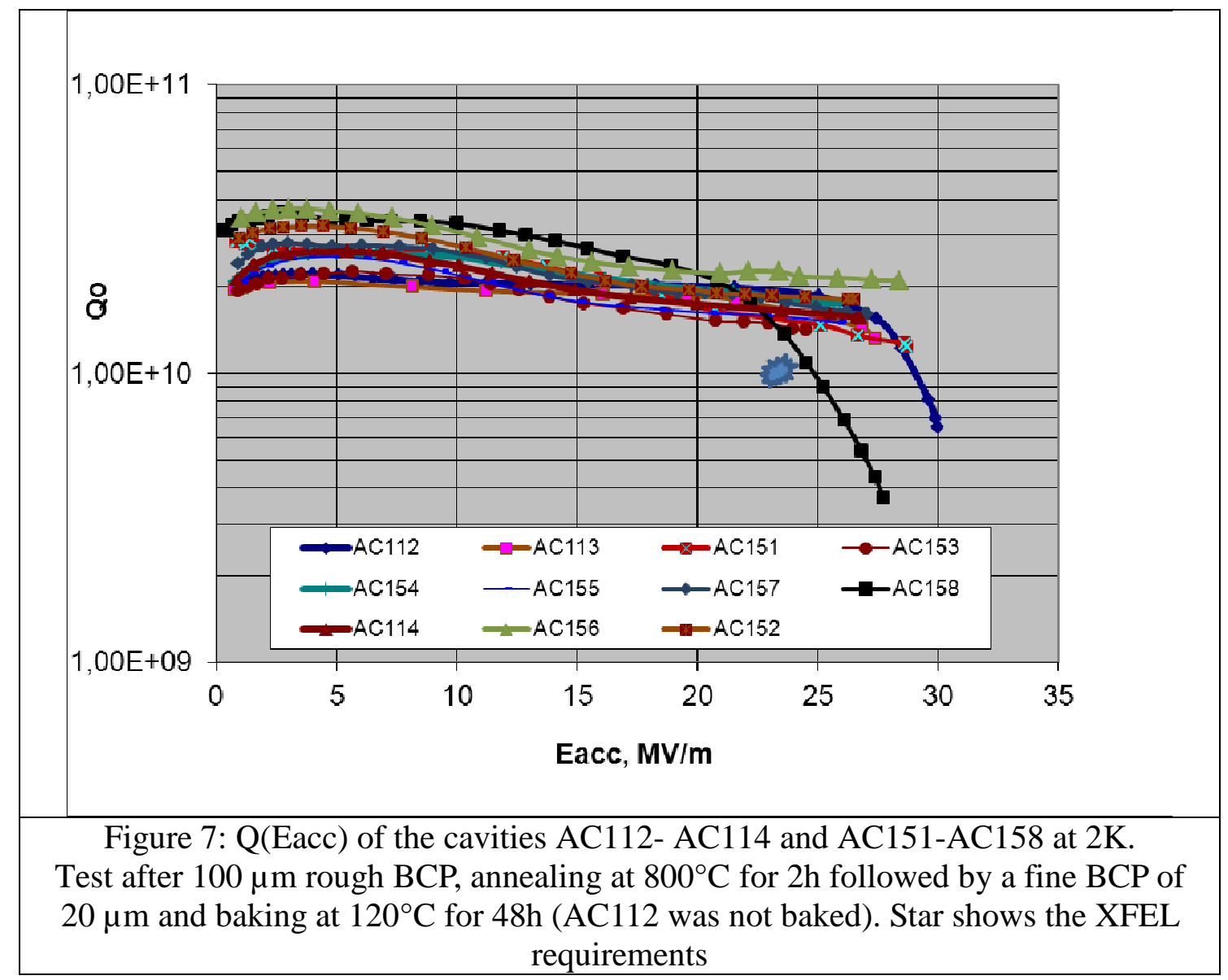

It is interesting to compare the performance of LG cavities with similar treated cavities from polycrystalline niobium (BCP treated and $800^{\circ} \mathrm{C}$ annealed). Several cavities have been treated that way in the scope of the TTF project at DESY in the 1990s. 


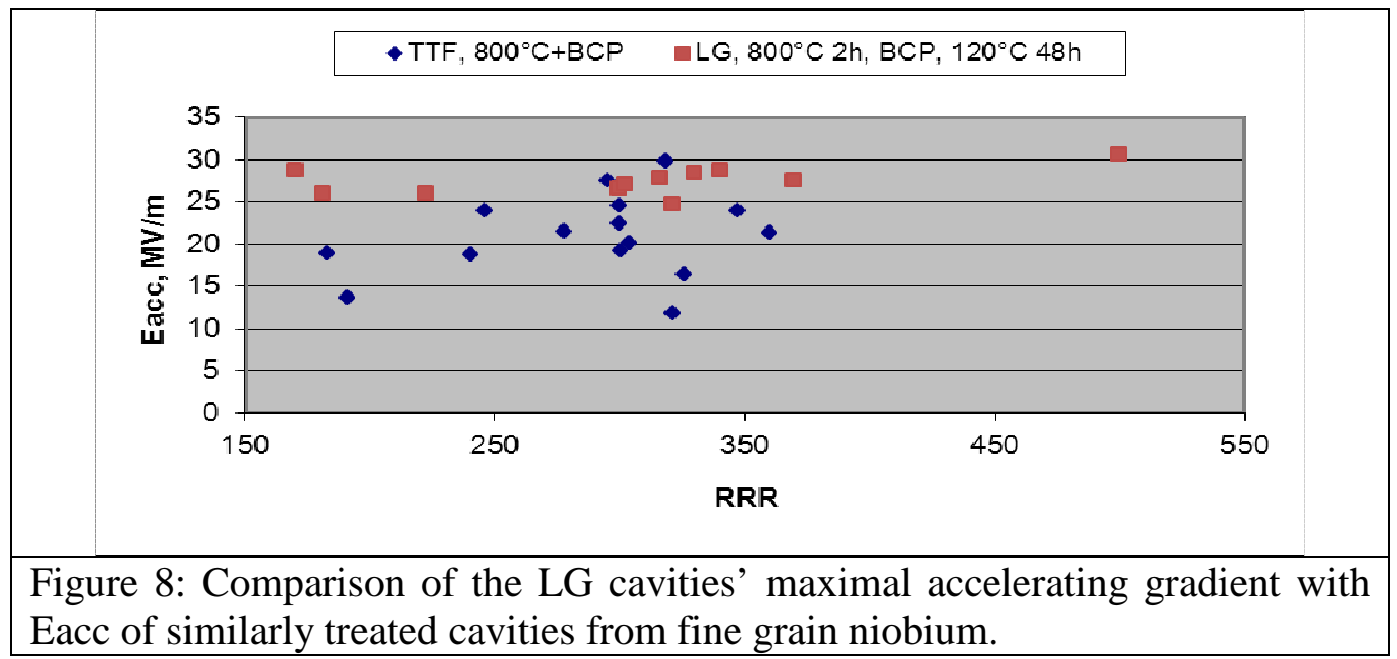

It can be seen (Fig.8) that the average value of Eacc for LG cavities is about $5-7 \mathrm{MV} / \mathrm{m}$ higher compared to the average Eacc of conventional cavities. Plausible reasons for such differences could be the difference in the thermal conductivity caused by phonon peak, or the mirror-like shiny internal surface of LG cavities [9].

Another interesting aspect of LG cavity behavior that has to be stressed is the rather high Qo even at high gradients after BCP treatment. As it can be seen in Fig. 9 the Qo reaches $3-6$ x $10^{10}$ at $1.8 \mathrm{~K}$ at moderate accelerating gradients. This value is almost a factor of two higher than for conventional fine grain cavities and indicates the high potential of LG cavities for continuous wave (CW) application.

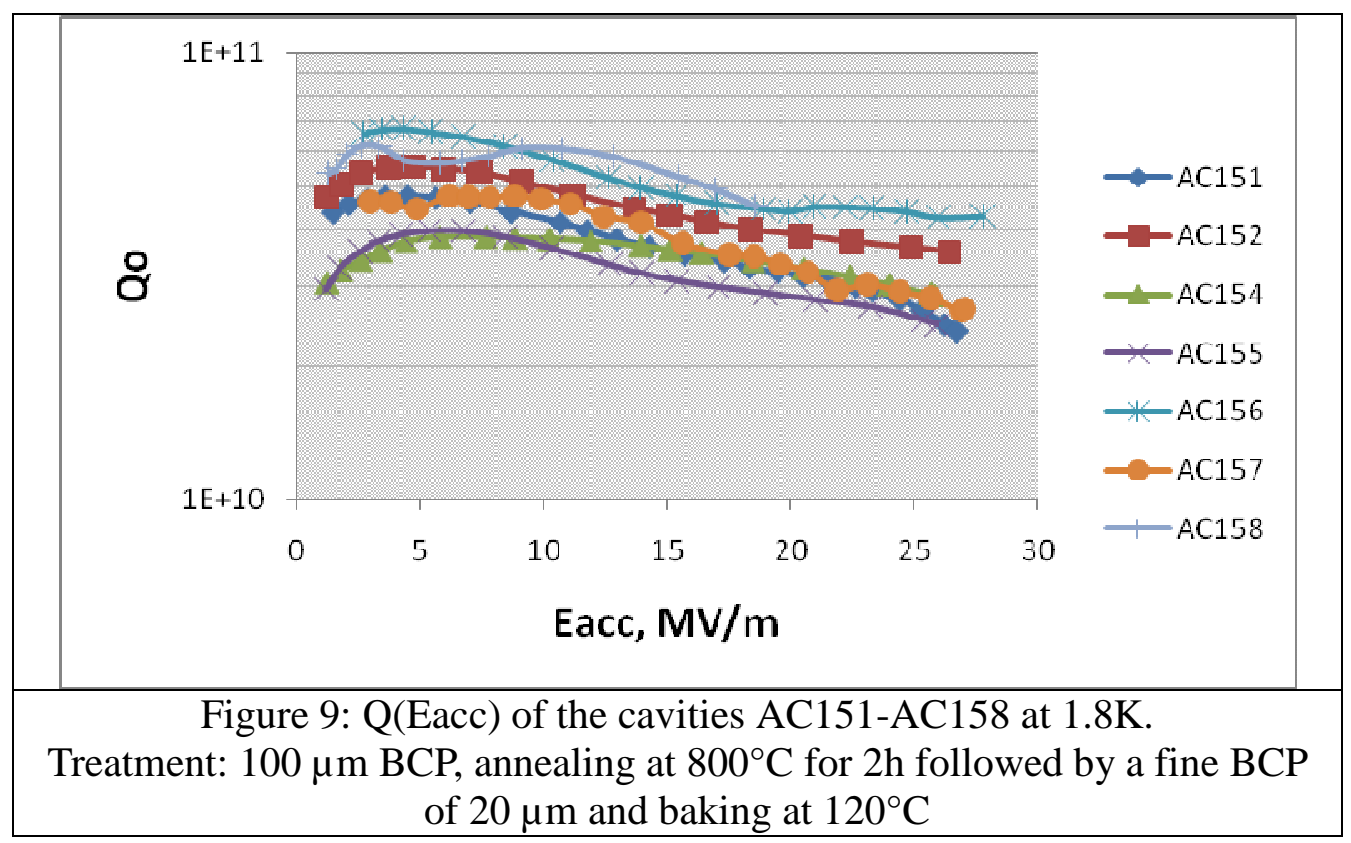

Comparision of EP and BCP treatment done on a few large grain single cell cavities has shown that EP works more efficiently. An increase of about $10 \mathrm{MV} / \mathrm{m}$ was achieved as an additional benefit during EP treatment on some previously BCP treated cavities [3]. 


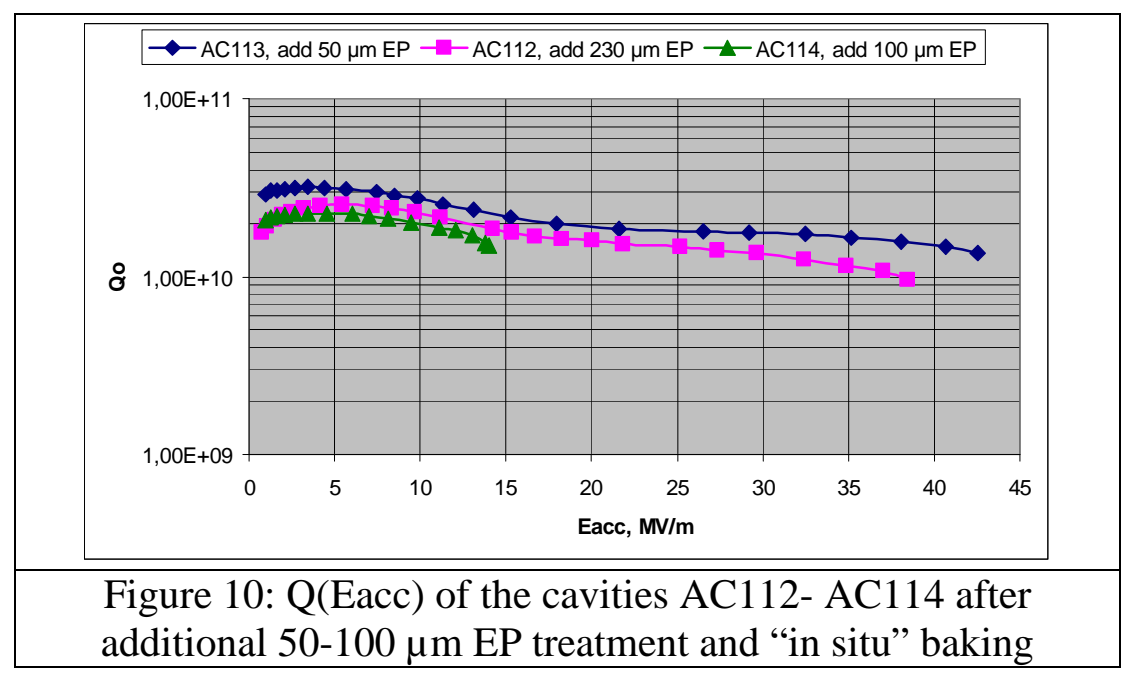

Therefore, as a next step, additional EP of approx. 50-100 $\mu \mathrm{m}$, combined with additional "in situ" baking, was applied to so far three LG 9-cell cavities AC112-AC114. For the AC112 cavity, several EP steps of 40-50 $\mu \mathrm{m}$ were done because of field emission. The resulting $\mathrm{Q}($ Eacc) excitation curves are shown in Fig. 10. Enhancement of the accelerating gradient by approximately $10 \mathrm{MV} / \mathrm{m}$ is observed for two cavities (AC112 and AC113).

Surprisingly, significant degradation of Eacc from 28 to $14 \mathrm{MV} / \mathrm{m}$ was observed for cavity AC114. Mode measurement has shown that the accelerating gradient of the individual cells was dramatically reduced too. After BCP treatment the Eacc was $28-35 \mathrm{MV} / \mathrm{m}$, but after EP the values were between 15 and $22 \mathrm{MV} / \mathrm{m}$. In order to check the quench position, an additional RF test was done on this cavity with a rotating T-map after 6 times HPR.

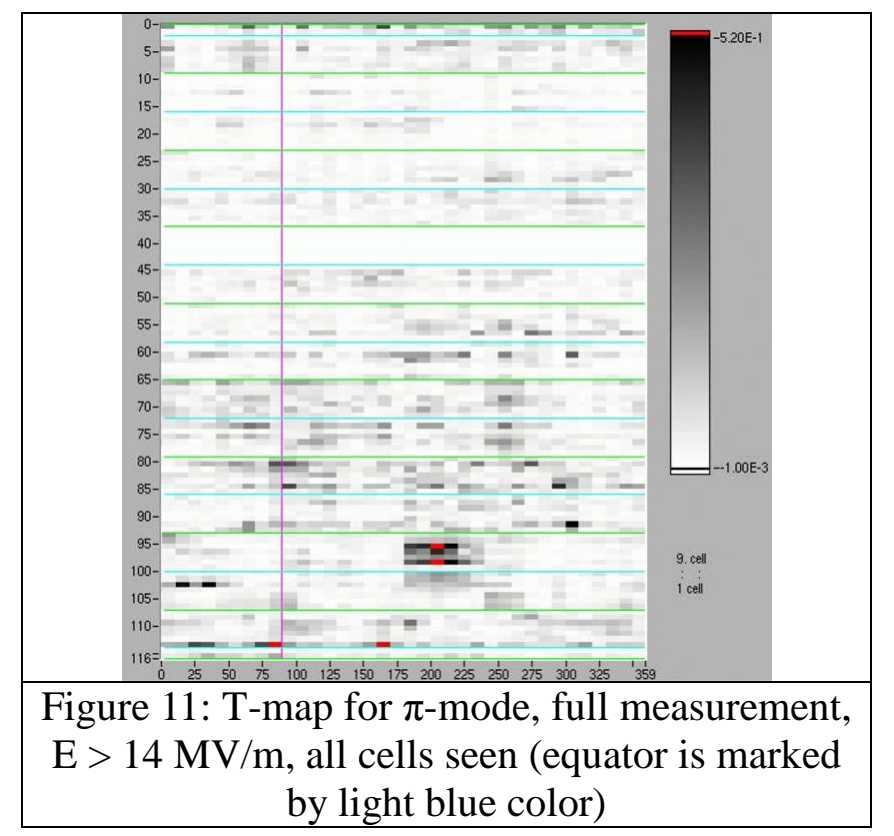

Local heating distribution registered by T-mapping is illustrated in Fig. 11. The same accelerating gradient of Eacc=14.1 MV/m, limited by thermal breakdown without field emission or multipacting as in previous tests, has been observed. T-maps were taken for all modes; for the $\pi$-mode, the quench was found in cell 2 near the equator. In other modes, quench was also found in cell 1 and 9. In addition, many other smaller hot spots were found by T-mapping (Fig. 11). 
The T-map results could be compared with optical inspection data using a high resolution camera available at DESY. Unfortunately, for the main hot spot in the cell 2 near the equator that caused the quench no clear picture could be obtained, due to the difficult lighting situation. Large groups of small craters on the entire inner and outer surface of all cells have been found. It appears that the craters may be responsible for performance degradation in all cells. Similar craters were discovered in the fine grain cavity Z110 (with similar Eacc) that correlated with the quench location detected by the T-map [10]. The cause of such craters is now being discussed, not only at DESY, but worldwide. It is difficult to say whether or not the LG grain surface behaves in an unusual manner during EP treatment compared to fine grain. It is possible that some imperfections (roots) were already present before EP and became larger with EP, if EP does not work properly. In any case, it is well known that EP works much better when starting with a previously prepared surface (the smoother the better). In light of this, large steps on grain boundaries of LG cavities can cause trouble for EP. There is not enough data in order to draw a conclusion concerning EP on 9 - cell cavities. For more statistics, all other available eight LG cavities AC151-AC158 are foreseen for electropolishing at DESY beginning in 2011.

The aim of the LG cavity development program is to pass all steps of the chain from material until integration into the helium tank and installation into the cryomodule for a test with electron beam in the accelerator. Up to now two LG cavities AC112 and AC113 reached the final stage of testing. Integration into the helium tank of these cavities was done routinely in the same way as for the fine grain XFEL prototype cavities; this did not produce any problems. Cavity AC112 is integrated into accelerator module $3 * * *$ and is currently working in the FLASH accelerator on position ACC1 (first module in the machine), operated at an accelerating gradient of $33 \mathrm{MV} / \mathrm{m}$. This accelerating gradient is limited by available RF power and not limited by the cavity itself. Cavity AC113 is installed in the accelerator module PXFEL1. Unfortunately in the horizontal Chechia test a degradation of Eacc from $36.8 \mathrm{MV} / \mathrm{m}$ to $27.5 \mathrm{MV} / \mathrm{m}$ was observed. After installation into the cryomodule the accelerating gradient remained unchanged. Accelerating module PXFEL1 including cavity AC113 is currently operated in the FLASH accelerator on position ACC7 (last module in the machine). Figure 12a \& b illustrates the position and performance of the LG cavities AC112 and AC113 in the accelerator modules of FLASH.

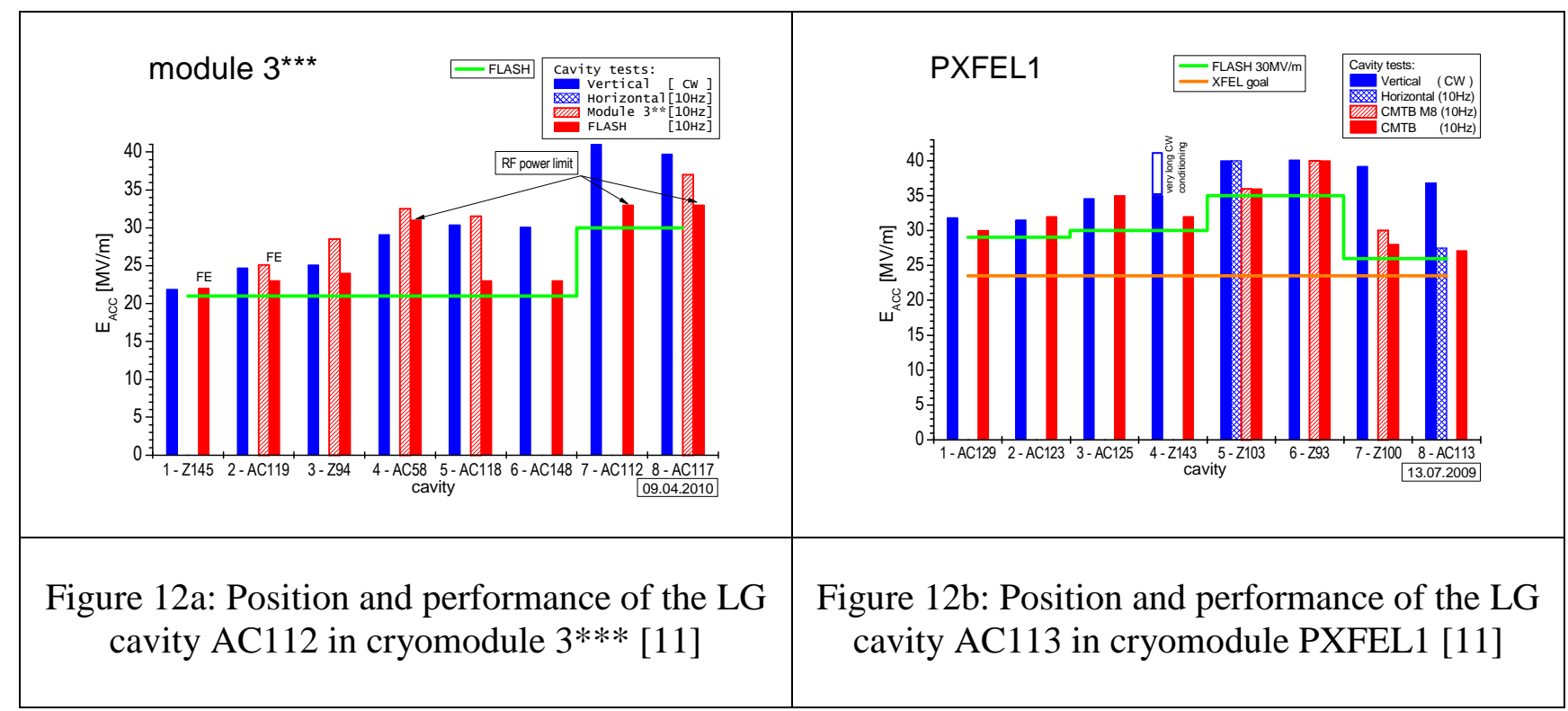




\section{SUMMARY}

Advantages of large grain cavities compared to fine grain were reported already several times and a summary was given in [14].

In this work it was shown that 9-cell cavities can be built from LG material without significant difficulties or special problems. Performance on the level of $25-30 \mathrm{MV} / \mathrm{m}(\mathrm{Bp}=110-130 \mathrm{mT})$ can be achieved in a stable and reproducible manner with BCP treatment in LG TESLA shape cavities. Accelerating gradients on the level of $40 \mathrm{MV} / \mathrm{m}$ can be achieved in TESLA shape LG cavities applying EP treatment.

The complete chain of the LG cavity technique beginning with material production and ending with cavity installation into a cryomodule was successfully tested. Cavity AC112 was installed into cryomodule $3 * * *$, cavity AC113 was installed in cryomodule PXFEL1 of the FLASH accelerator at DESY. Both cavities are currently working in FLASH and contribute to the beam electron energy of $1.25 \mathrm{GeV}$ and wave length of $4.12 \mathrm{~nm}$, which allows for achieving the so-called water window wavelength region and thereby creates excellent conditions for FLASH users [15] .

Unfortunately at the moment the industry is still not in position to produce in rather short time (1.5 years) the required amount of ca. 20 tons of LG material for the European XFEL. Nevertheless there is no doubt that the high potential of LG cavities will be taken into consideration for future accelerators. Considering the high quality factor Qo, simple preparation procedure and small scattering in the performance, BCP treated LG cavity can be recommended for application in accelerators with medium requirements for accelerating gradients, in particular for $\mathrm{CW}$ operations. The LG BCP option can be reasonable even for the ILC-project, this with a perhaps changed cavity shape. For example Low Loss (LL) shape allows lower cavity magnetic to electric field ratio by ca. $15 \%$ and enhance the limit in the achievable accelerating gradient [16].

\section{ACKNOWLEDGEMENTS}

We would like to thank our colleagues A. Ermakov, B. van der Horst, D. Kostin, D. Reschke, L. Lilje and W.-D. Moeller for their support of this work

\section{References}

[1] P.Kneisel, G.R.Myneni, G.Giovati, J.Sekutowicz and T.Carneiro. Development of Large grain/Single Crystal Niobium Cavity Technology at Jefferson Lab. International Niobium Workshop, October 30 - November 1, 2006, Araxa, Brazil. AIP Proceedings, New York, 2007, p. 84-97

[2] P. Kneisel, "Progress on Large Grain and Single Grain Niobium", SRF07, Beijing, Oct. 2007, TH102.

[3] W. Singer et al. Advances in Large Grain/Single Crystal SC Resonators at DESY. PAC'07, Albuquerque, USA, June 25-29, 2007, Paper THOAKI01

[4] K. Saito, F. Furuta, and H. Umezawa, "Multi-wire Slicing of Large Grain Ingot Material", in Proceedings of the $14^{\text {th }}$ International Conference on RF Superconductivity, Berlin, Germany, 2009, pp. 467-472.

[5] W. Singer et al. International Niobium Workshop, October 30 - November 1, 2006, Araxa, Brazil. AIP Proceedings, New York, 2007, p.123-132.

[6] G. Kreps, D. Proch, J. Sekutowicz. Half-cell and Dumb-bell Frequency Testing for the Correction of the TESLA Cavity Length. Proceedings of 9th Workshop on RF Superconductivity. Santa Fe, New Mexico, USA, 1999, p. 499-504 
[7]. W. Singer, S. Aderhold, J. Iversen, G. Kreps, L. Lilje, A. Matheisen, X. Singer, H. Weise, M. Pekeler, J. Schwellenbach, F. Scholz, B. Spaniol, E. Stiedl, Development of large grain superconducting resonators for the European XFEL, 23rd PAC'09, May 4-8, 2009, Vancouver, Canada, Paper TU5PFP054

[8] P. Kneisel, G. R. Myneni, G. Ciovati, J. Sekutowicz, and T. Carneiro, "Performance of Large Grain and Single Crystal Niobium Cavities" in Proceedings of the $12^{\text {th }}$ Workshop on RF Superconductivity, Ithaca, NY, 2005, paper MoP09

[9] A. Ermakov, I. Jelezov, X. Singer, W. Singer, G. B. Viswanathan, V. Levit, H. L. Fraser, H. Wen and M. Spiwek, Physical properties and structure of large grain/single crystal niobium for superconducting RF cavities // Journal of Physics: Conference Series, 97 (2008) 012014

[10]. X. Singer, S. Aderhold, A. Ermakov, W. Singer, K. Twarowski, M. Hoss, F. Scholz, B. Spaniol, Surface Investigation on prototype cavities for the European XFEL, IPAC 2010 (Kyoto, Japan), The First International Particle Accelerator Conference (23-28 May, 2010)

[11] D. Kostin, W.-D. Moeller, A. Goessel, K. Jensch, "Superconducting Accelerating Module Tests at DESY", in Proceedings of the 14th International Conference on RF

Superconductivity (SRF2009), September 20-25, 2009, Berlin, Germany, pp. 180-184. D. Kostin.

Private communication

[12] A. Dangwal Pandey, G. Mueller, D. Reschke, X. Singer, Field Emission from Crystalline Niobium, Phys. Rev. STAB 12 (2009) 2, 023501(0)-023501(7)

[13] TESLA Technical Design Report DESY Hamburg, March 2001.

[14] Gianluigi Ciovati, Peter Kneisel and Ganapati R. Myneni. America's Overview of Superconducting Science and Technology of Ingot Niobium. These proceedings.

[15] DESY NEWS. Hamburg 2010/09/28 . FLASH opens the view through the water window

[16] Jacek Sekutowitcz. Multi-cell Superconducting Structures for High Energy e ${ }^{+} \mathrm{e}^{-}$Colliders and

Free Electron Laser Linacs. EuCARD Editoral Series on Accelerator Science. Warsaw, 2008, 135 pages. 